\title{
Convalescent Plasma Therapy: Treatment Option for SARS-COV-2 Infection
}

\section{Aashi Jain ${ }^{1}$, Irfanul Haque ${ }^{2}$, Sarita Jangra Bhyan ${ }^{1}$, Besty Thomas ${ }^{1}$, Amrita Kumari $^{1}$, Kulsoom Hamid ${ }^{1}$, M. Sreelakshmi ${ }^{1}$, Nancy Goel ${ }^{1}$, Rashi Chauhan ${ }^{1}$}

1. Pharm D Intern Students, Department of Pharmacy Practice, Teerthanker Mahaveer College of Pharmacy, TMU, Moradabad, Uttar Pradesh, India. Head of Department, Department of Clinical Pharmacology Jaypee Hospital, Noida, Uttar Pradesh, India. Internship Coordinator, Assistant Professor, Department of Pharmacy Practice, Teerthanker Mahaveer College of Pharmacy, TMU, Moradabad, Uttar Pradesh, India.

*Corresponding author's E-mail: aashijain979@gmail.com

Received: 04-06-2020; Revised: 12-08-2020; Accepted: 19-08-2020.

DOI: $10.47583 /$ ijpsrr.2020.v64i01.029 \begin{abstract}
The number of corona virus cases is steeply increasing day by day worldwide. According to WHO, Severe Acute Respiratory Syndrome Coronavirus-2 (SARS-CoV-2) infection is managed by preventing infection, detection of cases, providing supportive care, and monitoring. No specific options (antiviral, monoclonal antibody or vaccine) are available for controlling and treatment of the COVID-19 infection, Convalescent plasma (CP) therapy becomes the first available option to treat this pandemic situation according to the successful use of plasma therapy in a historical corona virus outbreak. Plasma obtained from COVID-19 recovered patients is used as prophylaxis therapy or as a therapeutic option. Plasma collection, preparation, and administration requires a proper procedure to enhance its productivity. Preparation of plasma with adequate neutralizing antibody titer becomes challenging. $\mathrm{CP}$ therapy may provide antiviral and immunomodulatory effect to SARS-CoV-2 infected patients by controlling overactive immune system such as cytokine storm, Th1/Th17 ratio, and hypercoagulable state. Based on the available case studies data, the administration of CP is proven to be beneficial in treating critically ill patients of SARS-CoV-2 infection. Passive antibody transfusion may reduce viral load with no severe adverse reaction and provide passive immunity against the virus. However, further welldesigned clinical trials are needed to establish true clinical efficacy and safety of plasma therapy.
\end{abstract}

Keywords: Convalescent plasma therapy, severe acute respiratory syndrome coronavirus 2 (SARS-CoV-2), Coronavirus (COVID-19), neutralizing antibody, passive antibody therapy.

\section{INTRODUCTION}

orona virus (COVID-19) has become a potential threat to human health and the economy. According to the World Health Organization (WHO), this outbreak became a "public health emergency of international concern" on January 31, 2020, and declared as corona virus pandemic on March 11, $2020^{1}$. In December 2019, the first case of COVID-19 occurred in Wuhan, China, caused by a newly identified $\beta$-corona virus (SARS-CoV-2). Within 2 months, the infection had already spread to different countries across the world ${ }^{2}$. As of July 23, 2020, WHO has reported globally 15, 404,722 confirmed cases, including 631, 009 deaths. Whereas in India, totally confirmed cases are 1, 241, 654 including 29, 906 deaths. This increasing statistical data suggests that an urgent solution needed to combat this pandemic and loss of life.

SARS-CoV-2 is a single-stranded RNA virus that belongs to the Corona viridae family and is the causative pathogen of the COVID-19 ${ }^{3}$. It was found that the genetic sequence of SARS-CoV- 2 is $96 \%$ similar to the bat corona virus CoV RaTG13 which accounts for the zoonotic origin of SARSCoV-2 i.e. virus is transmitted to the humans from bat species ${ }^{4}$. The genomic sequence of SARS-CoV-2 has 4 major proteins i.e. spike protein (S), membrane protein $(\mathrm{M})$, an envelope protein (E), and nucleocapsid protein (N). Among these, spike
(S) protein is of most importance which provides the crown-like appearance to the SARS-CoV-2 and is responsible for the binding of SARS-CoV-2 with angiotensin-converting enzyme (ACE2) receptor of the host cell ${ }^{5}$. Therefore, this binding is responsible for the infection of the COVID-19.

COVID-19 is generally spread through the aerosol droplets or respiratory secretion of infected patients, contaminated objects, faecal swabs, or blood signifying a multiple routes of transmission of SARS-CoV- $2^{6}$.

According to epidemiological data, the incubation period of SARS-CoV-2 is 1-14 days and symptoms mostly occur within 3-7 days ${ }^{7}$. SARS-CoV-2 shares similar genetic features with severe acute respiratory syndrome corona virus (SARS-CoV) and the Middle East respiratory syndrome corona virus (MERS). Therefore, patients show identical clinical manifestations related to SARS-CoV and MERS. The most common clinical symptoms are mild to moderate $(80 \%)$ includes fever, dry cough, malaise, shortness of breath, diarrhoea, loss of taste or smell, and very few severe cases (14\%). Critical cases $(5 \%)$ require admission to the intensive care unit (ICU) who develops acute respiratory distress syndrome (ARDS), sepsis, shock, coagulation dysfunction, and multi-organ damage ${ }^{8,}$. SARS-CoV-2 is highly susceptible to elderly ( $>65$ years of age), male gender, obese (BMI $\geq 30)$, and people with underlying disease conditions (hypertension, diabetes, 
cardiovascular disease, chronic obstructive pulmonary disease) ${ }^{1}$.

There is currently no evidence that any pharmacological agent, vitamin, or herbal supplement is effective in the prevention of COVID-19. Several prevention trials are ongoing to stop the chain of COVID-19. A few nonpharmacological steps to prevent the spread of disease are washing hands, maintaining social distance and hygienic environment, wearing masks, and personal protective equipment (PPE) for healthcare workers and pharmacies.

Pharmacological choices include acetaminophen, ibuprofen, aspirin, and naproxen as antipyretic agents. Overall, there is little evidence for or against the effectiveness of the non-prescription cough medication such as antitussive (dextromethorphan) or expectorants.

Experimental therapies 10 for COVID-19 include azithromycin in combination with hydroxychloroquine for potential synergistic antiviral effect and to prevent concurrent bacterial infections (e.g. pneumonia). Chloroquine (Antimalarial) reduces exacerbations of pneumonia, shortening disease course and decrease viral load. Janus kinase (JAK1 and JAK2) inhibitor (Baricitinib and ruxolitinib) reduces hyperinflammatory (cytokine storm syndrome) complications. Corticosteroids (immunomodulator) have shown a positive effect on mortality and resolution of shock. Lopinavir/ ritonavir (antiretroviral) has activity against SARS-CoV-1, MERS$\mathrm{CoV}^{11}$. Remdesivir (antiretroviral) has been reported to treat the first US case of COVID-19 successfully ${ }^{12}$. In-vitro studies have proved the use of remdesivir and chloroquine combination to inhibit the recently emerged SARS-CoV-2 infection. Tocilizumab (IL-6 monoclonal antibody) reduces complications due to hyper inflammation.

Another therapeutic option to treat COVID-19 patients is convalescent plasma therapy or immunoglobulin. Several studies have been started in different parts of the world to test the effectiveness of this therapy in the critical cases of COVID-19. Therefore, this article will summarize the method of administration, preparation of the convalescent plasma and its therapeutic role in COVID-19.

\section{Plasma Therapy}

Convalescent plasma therapy (CP) is considered as one of the historical and classical treatment to control the mortality rate in a critically ill patient due to the COVID-19 pandemic. It is an approach of passive immunization that has been used in preventing and controlling of infectious diseases (SARS, influenza A/H5N1, Ebola) since the early 20th century ${ }^{13}$. Convalescent plasma therapy $(C P)$ is the administration of passive antibodies against a target organism to a predisposed individual for the purpose of controlling and preventing infectious disease and to provide immediate immunity against the infection in the susceptible individual.

\section{Chronological Perspective of Plasma Therapy}

In 1890, German physiologist Emil Von Behring was the first to discover the plasma therapy ${ }^{14}$. During the Spanish flu pandemic in 1918, this treatment became effective in reducing mortality and fatality rate. Luke and colleagues conducted a meta-analysis of eight studies from 1918 to 1925 by involving 1703 subjects who received influenzaconvalescent infusion which resulted in a reduction of $21 \%$ fatality rate ${ }^{15}$.

The use of plasma treatment in diphtheria (1920) and avian influenza A (H5N1) (2003) outbreak was proven to be beneficial by reducing invasive ventilation and respiratory viral load. During 2003 SARS-CoV outbreak, a non-randomized study conducted in Hong Kong where 50 subjects were administered with a plasma collected from the SARS-CoV recovered donor and showed improvement in fatality rate, significantly reducing the day of hospitalization by 22 days when compared to the other group who were taking steroids ${ }^{16}$.

In 2009, a Cohort study by Hung and colleagues in influenza A H1N1 showed significant reduction of viral load after plasma therapy administration and decreased mortality rate within 5 days of symptoms onset ${ }^{17}$. During the Ebola outbreak in 2014, the use of plasma therapy was recommended by WHO as an empirical therapy however, this therapy may result in few ADRs such as nausea, fever, and skin erythema ${ }^{18}$. The safety and efficacy of the CP have been historically established in the epidemic situation of influenza A, SARS-CoV, and MERS.

Therefore, the use of convalescent plasma from Spanish influenza to the existing pandemic was proven to be effective in reducing fatality rate and hospital stay. Due to the efficacy and tolerability in previous outbreaks, CP is considered as a potential therapeutic choice in treating COVID-19 patients.

\section{Protocol for Collection, Preparation, and administration of Convalescent Plasma}

Apheresis is the standard procedure for obtaining plasma. This process involves continuous centrifugation of a donor's blood to collect the plasma ${ }^{19}$. Following conditions and elements need to be fulfilled before deploying plasma such as $20,21,23$.

a) Donor availability- those who have been fully recovered from the disease and are ready to donate plasma. Eligible donor having age between 18-65 years with a negative test for COVID-19 after 14 days of recovery. These tests are repeated after 48 hours and at the moment of plasma donation ${ }^{22,23}$.

b) A blood bank facility, virology laboratory to facilitate the plasma donation.

c) Plasma obtained must be of high quality i.e. it must be free from any infection (HIV, hepatitis A, and $B$, human T-cell lymphotropic virus 1 and 2 , 
syphilis). Therefore, screening of recovered COVID-19 donors is the next step. Viral nucleic acid and antibody screening are the recommended standardized test. ${ }^{23,24}$.

Another procedure to improve the safety of plasma is inactivation of the organism with psoralen or riboflavin along with ultraviolet exposure.

d) Serological assay and virology assays are recommended to detect SARS CoV- 2 and to measure viral neutralization respectively ${ }^{25}$.

e) Desired level of antibody selection is necessary. FDA has recommended high neutralizing antibody titers (>1:320 for India) ${ }^{26}$.

f) Sufficient amount (around $400-800 \mathrm{ml}$ ) of plasma is to be collected and processed into specific product e.g. fresh frozen plasma, fresh or lyophilized plasma.

g) The plasma is stored in units of 200 or $250 \mathrm{ml}$ and the registry is maintained for further use.

Plasma contains a wide variety of blood components, a mixture of inorganic and organic salts, water, more than 1000 proteins such as albumin, immunoglobulin, coagulating agents, antithrombotic agents, and many others ${ }^{27}$.

Although, a standard neutralizing titer for transfusion of plasma is not well established. A donor is selected for higher antibody titer but the neutralizing titers differ according to the patient's character. Therefore, optimization of plasma preparation according to patient's need becomes necessary for prophylactic and therapeutic dosing ${ }^{20}$. Till now we do not know the effective neutralizing antibody titers and administration timing of plasma in COVID-19 patient for prophylactic and therapeutic dosing. Hence, to determine this parameter, clinical trials need to be conducted.

\section{Antiviral and Immunomodulatory Effect of Plasma Therapy}

It is assumed that convalescent plasma transfusion will provide an antiviral and immunomodulatory effect in the COVID-19 patient which helps in eradication of cytokine storm. Neutralizing antibody is responsible for reducing viral load and decreasing the infection. Therapeutic effect of CP depends upon the concentration of the neutralizing antibody titers. Although, its effect may also be enhanced by activation of antibody-mediated pathways such as cellular cytotoxicity, complement system, or phagocytosis 28. Previous data from SARS-CoV and MERS demonstrated that neutralizing antibody attached to the spike (S1 and S2) protein of the viral molecule inhibited the viral entry replication and thereby suppressing the infection ${ }^{29}$.

Apart from neutralizing antibody, other antibody such as immunoglobulin (IgG and IgM) plays a protective role in COVID -19. These non-neutralizing antibodies may not interfere with viral replication and as a result is used as a prophylactic therapy ${ }^{19}$.

Immunoglobulins IgG is produced against nucleoprotein (N) in SARS-CoV infection and its level is detected on day 4 of symptom onset ${ }^{30}$. A higher concentration of IgM and IgG levels was detected at day 9 and the second week of symptom onset, respectively ${ }^{31}$.

In COVID-19 infection, the immune system becomes hyperactive and starts overproducing inflammatory cytokines, Th1, and Th17and other inflammatory agents. The immunomodulatory effect of plasma involves the network of autoantibody which provides antiinflammatory action and controls the overactive immune system i.e. complement system, Th1/Th17 ratio, cytokine storm, and regulation of hypercoagulable state ${ }^{28}$.

\section{Evidences of Plasma Therapy use in COVID-19}

Few studies which demonstrated the use of plasma therapy in COVID-19 patient and its outcome:

a. Zhang B and colleagues conducted a study on four critical patients who were treated with supportive care along with CP. All four SARSCoV-2 patients (including pregnant females) were recovered with no serious adverse reaction. Three patients were tested for either immunoglobulin (IgM and IgE) or viral load. In first case, viral load was significantly decreased after CP infusion. In third and fourth cases, antiSARS-CoV IgG are produced after 14 days of the CP therapy. All four patients showed a negative SARS-CoV-2 test within 3-22 days ${ }^{32}$.

b. Jin Young Ahn and colleagues conducted a study in South Korea of 71 year old male patient who was administered $500 \mathrm{ml}$ of CP in 2 doses at $12 \mathrm{~h}$ of interval on the $10^{\text {th }}$ day of admission along with antiviral and hydroxychloroquine. Severe acute respiratory distress syndrome was observed during CP transfusion and the patient was on mechanical ventilation. The outcome of the therapy indicated that the viral load changed from day $10^{\text {th }}(24.98)$ to $26^{\text {th }}$ day (negative) and the patient weaned from mechanical ventilation 33 .

c. Mingxiang Ye in China conducted a study on CP transfusion to 69 year old male, administering $600 \mathrm{ml}$ of CP in 3 doses on the $33^{\text {rd }}$ day of symptom onset. The concomitant drug was arbidol and levofloxacin. Patient was feeling Myalgia and chest CT showed patchy areas of GGOs at the time of transfusion. After CP therapy, patient symptoms were improved. Viral load became negative and GGOs were resolved on the $37^{\text {th }}$ day. No severe adverse reactions were observed ${ }^{34}$.

d. Shen et al, presented a study on 5 critically ill patients, administering $200 \mathrm{ml}$ of plasma in 2 
doses within 10-22 days with antibody titer $>1: 1000$. The result suggested that the body temperature was normalized within 3 days in 4 out of 5 patients. SOFA score decreased, $\mathrm{PAO}_{2} /$ $\mathrm{FIO}_{2}$ level increased, viral load decreased and became negative within 12 days. ARDS was resolved at day 12 in 4 patients ${ }^{35}$.

e. Duan and colleagues conducted a study in china on 10 ( 6 male and 4 female) severally ill patients (median age 52.5year). Out of 10, four patients had a chronic illness (cardiovascular, HTN, and cerebrovascular disease). $200 \mathrm{ml}$ of plasma was transfused to each patient within 16.5 days (median value) antibody titer of $>1: 640$. These patients were on other antiviral, antibacterial, and antifungal therapy. All patients were in ICU and 3 patients on mechanical ventilation. Within 3 days of plasma transfusion, clinical symptoms were improved, oxyhemoglobin saturation increased whereas viral load and inflammation decreased. Two patients were weaned from mechanical ventilation. No severe adverse effect was noticed. However, one patient had a facial red spot. Three patients were discharged and seven patients showed improvement in their condition ${ }^{36}$.

\section{Risk and Benefits of Plasma Transfusion}

Plasma therapy may be either used as prophylaxis or therapeutic treatment. In case of the prophylactic treatment, CP provides immunity against SARS-CoV-2 and also helps in preventing COVID-19 infection in those who are more vulnerable and in close contact with the confirmed cases for example healthcare workers. Whereas, in case of therapy, antibody transfusion is most effective when administered shortly after the onset of symptoms. Therapeutic treatment with $\mathrm{CP}$ is used to reduce symptoms and viral load of SARS-CoV-2 ${ }^{21}$. Clinically, CP is used for prophylactic treatment in rabies, hepatitis $B$, and severe respiratory syncytial virus (RSV) disease ${ }^{37}$. Hence, historical studies proved the use of $C P$ as a prophylactic treatment is more effective than the therapeutic treatment of an infectious disease.

Risk assessment of the administration of plasma therapy is categorized into two categories

a) Known risk: occur during blood substance transfusion, which happens due to accidental infection with other disease agents causing reactions to serum components and immunological reaction for example serum sickness. Commonly, pulmonary disease is therapeutically treated with $\mathrm{CP}$, in which plasma infusion may carry a risk of transfusion-related acute lung injury (TRALI). In this situation, riskbenefit assessment is required ${ }^{38}$.

b) Theoretical risk: it involves antibody- dependant enhancement of infection (ADE). ADE phenomenon occurs in many viral infections resulting in enhancement of the disease in the occurrence of certain antibodies. An example of such is the administration of antibodies to SARSCoV-2 exposed individuals may prevent disease in such a manner that weakens the immune response by leaving the person susceptible to future infection. Clinical trials are needed to measure such findings. If the threat is confirmed, then as soon the vaccine becomes available, these individuals could get a vaccination against COVID-19. ${ }^{14}$

\section{Safety and Efficacy Evaluation}

Recent data of plasma transfusion in COVID-19 patient has not reported any serious adverse drug event 22,36 . Although, the rate of infusion should be slow under proper monitoring to identify any transfusion-related side effects. Close monitoring is also required after transfusion to recognize any ADRs especially related to inflammatory changes of lungs or systematically ${ }^{22}$.

The efficacy of the plasma therapy depends upon the concentration of antibody administered; hence a sufficient amount of antibody must be administered to a susceptible person. These antibodies circulate in the blood, reach to tissues and provide protection against infection. In-vivo trials suggest that the use of the plasma will not only clear the viral load or prevent new infection, but also helps in the clearance of the infected cells ${ }^{39}$. The primary immune response of plasma administration occurs within 10-14 days, followed by viral load clearance. A viral infection is at peak level during the first week of infection, therefore administration of the plasma at an early stage becomes more beneficial to the high-risk individual (age is above 70 or under less than $94 \%$ oxygen saturation). Recent data suggest that the administration of CP within 5 days of disease onset would be the most appropriate option. The primary aim for transfusion of antibody therapy is to increase survival rate and the secondary aim is the decreased hospital stay and improvement in the patient's condition ${ }^{23}$.

One study indicated that no adverse effect was observed in the patient with CP administration. Six patients were in the treatment group and all showed viral clearance whereas 15 patients were in the control group. 5 patients from the treatment group and 14 patients from the control group died $(P=.18)$. Recovery rate is one from each group. Before death, 5 patients (100\%) from treatment group and 3 out of 14 patients from the control group showed undetectable SARS-CoV-2 ( $\mathrm{P}=$ $.005)$. The rate of survival is more in the treatment group compared to the control group $(P=.03)^{40}$.

\section{Limitation of the Plasma Therapy}

Previous data showed that the administration of plasma for the reduction of viral load is poorly controlled with limited data ${ }^{16}$. Hence, large scale randomized clinical trials are needed to establish a true clinical outcome of 
the plasma transfusion. Furthermore, the true efficacy of this treatment is not evaluated since patients are taking other therapies (antiviral, immunomodulator, and steroids) along with plasma therapy ${ }^{14}$. The selection of standard neutralizing antibody titer for each patient was not established. Large scale studies are required to investigate the optimal timing of CP transfusion ${ }^{41}$. These are the few limitations that need to be resolved for better safety and efficacy of the plasma therapy.

\section{CONCLUSION}

In the absence of definitive treatment for the COVID-19 pandemic, infusion of plasma therapy is proven to be beneficial in improving clinical deterioration, decreased viral load, and extend the survival rate in critically ill patients. No severe adverse effects were noticed in plasma therapy studies yet. Although, proper randomized clinical trials are necessary for evaluating the further safety and efficacy of the plasma transfusion in COVID-19.

Acknowledgment: The author would like to thank the institution and colleagues for their enormous support and encouragement.

\section{REFERENCES}

1. Zhou M, Zhang X, Qu. J. Corona virus disease 2019 (COVID19): a clinical update. Front. Med. 14(2), 2020, 126-135.

2. Guo Y.R., Cao .Q.D. The origin, transmission and clinical therapies on corona virus disease 2019 (COVID-19) outbreak - an update on the status. Military Medical Research.7, 2020, 11.

3. Zhu N, Zhang D, Wang W, Li X, Yang B, Song J. A novel corona virus from patients with pneumonia in China, 2019. N Engl J Med. 382(8), 2020, 727-33.

4. Chen N, Zhou M, Dong X, Qu J, Gong F, Han Y . Epidemiological and clini- cal characteristics of 99 cases of 2019 novel corona virus pneumonia in Wuhan, China: a descriptive study. Lancet. 395(10223), 2020, 507-13.

5. Forni $D$, Cagliani R, Clerici M, Sironi M. Molecular evolution of human corona virus genomes. Trends Microbiol. 25(1), 2017, 35-48.

6. Zhang W, Du RH, Li B, Zheng XS, Yang XL, Hu B. Molecular and serological investigation of 2019-nCoV infected patients: implication of multiple shedding routes. Emerg Microbes Infect. 9(1), 2020, 386-9.

7. Jin $\mathrm{YH}$, Cai L, Cheng ZS, Cheng H, Deng T, Fan YP. A rapid advice guideline for the diagnosis and treatment of 2019 novel corona virus (2019-nCoV) infected pneumoni (standard version). Mil Med Res. 7(1), 2020, 4.

8. Poutanen SM, Low DE, Henry B, Finkelstein S, Rose D, Green $K$. Identification of severe acute respiratory syndrome in Canada. N Engl J Med. 348(20), 2003, 1995-2005.

9. Huang $C$, Wang $Y$, Li X, Ren L, Zhao J, Hu Y. Clinical features of patients infected with 2019 novel corona virus in Wuhan, China. Lancet. 395(10223), 2020, 497-506.

10. Wang L, Wang Y, Ye D, Liu Q. Review of the 2019 novel corona virus (SARS-CoV-2) based on current evidence. International Journal of Antimicrobial Agents. 2020, 1-7.
11. Li H, Wang YM, Xu JY, Cao B. Potential antiviral therapeutics for 2019 Novel Corona virus. Chin J Tuberc Respir Dis. 3(0), 2020, E002.

12. Holshue ML, DeBolt C, Lindquist S, LofyKH, Wiesman J, Bruce H. First case of 2019 novel corona virus in the United States. N Engl J Med. 2020.

13. Marano G, Vaglio S, Pupella S, Facco G, Catalano L, Liumbruno GM. Convalescent plasma: New evidence for an old therapeutic tool? Blood Transfuse. 14, 2016, 152-7.

14. Muhammed Anas. Role of Convalescent Plasma Therapy in Critically III Corona virus Disease-19 (COVID-19) Patients. EC Pulmonology and Respiratory Medicine. 9(6), 2020, 50-56.

15. Luke TC, Kilbane EM, Jackson JL, and Hoffman SL. Metaanalysis: convalescent blood products for Spanish influenza pneumonia: a future H5N1 treatment? Ann Intern Med. 145, 2006, 599-609.

16. Mair-Jenkins J. The effectiveness of convalescent plasma and hyperimmune immunoglobulin for the treatment of severe acute respiratory infections of viral etiology: a systematic review and exploratory meta-analysis. The Journal of Infectious Diseases. 211(1), 2015, 80-90.

17. Hung I.F.N, To K.K.W, Lee C.K. Hyperimmune IV immunoglobulin treatment: a multicenter double-blind randomized controlled trial for patients with severe 2009 influenza A (H1N1) infection. Chest. 144, 2013, 464-73.

18. Van Griensven J, Edwards T, de Lamballerie X, Semple MG. Evaluation of convalescent plasma for Ebola virus disease in Guinea. N Engl J Med. 374, 2016, 33-42.

19. Bloch EM, Shoham S, Casadevall A, Sachais BS, Shaz B, Winters JL. Deployment of convalescent plasma for the prevention and treatment of COVID- 19. J Clin Invest 2020.

20. Bethany L, Brown, Jeffrey Mc Cullough. Treatment for emerging viruses: Convalescent plasma and COVID-19. Transfusion and Apheresis Science. 59(3), 2020, 102790.

21. Casadevall A, Pirofski LA. The convalescent sera option for containing COVID-19. J Clin Invest. 130(4), 2020, 1545-1548.

22. Duan K, Liu B, Li C, Zhang H, Yu T, Qu J. Effectiveness of convalescent plasma therapy in severe COVID-19 patients. Proc Natl Acad Sci USA 2020.

23. Tiberghien $P$, de Lambalerie $X$, Morel $P$, Gallian $P$, Lacombe $K$, Yazdanpanah Y. Collecting and evaluating convalescent plasma for COVID-19 treatment: Why and how. Vox Sang 2020.

24. Dodd RY, Crowder LA, Haynes JM, Notari EP, Stramer SL, Steele WR. Screening blood donors for HIV, HCV, and HBV at the American Red Cross: 10-year trends in prevalence, incidence, and residual risk, 2007 to 2016. Transfus Med Rev 2020.

25. Okba NMA, Widjaja I, Li W. Serologic detection of middle east respiratory syndrome coronavirus functional antibodies. Emerg Infect Dis. 26(5), 2020.

26. Center for Biologics Evaluation and Research. Investigational COVID-19 convalescent plasma - emergency INDs 26 March 2020. Available at: https://www.fda. gov/vaccines-bloodbiologics/investigational-new-drug-ind-or-deviceexemptionide-process-cber/investigational-covid-19convalescent-plasma-emergency-inds. 
27. Benjamin RJ, McLaughlin LS. Plasma components: Properties, differences, and uses. Transfusion. 52(Suppl. 1), 2012, 9S-19S.

28. Rojasa M, Rodrígueza Y, Diana M. Monsalvea, Ampudiaa Y A, Camachoc B. Convalescent plasma in Covid-19: Possible mechanisms of action. Autoimmunity Reviews. 19(7), 2020, 102554.

29. Du L, He Y, Zhou Y, Liu S, Zheng B-J, Jiang S. The spike protein of SARS-CoV-a target for vaccine and therapeutic development. Nat Rev Microbiol. 7, 2009, 226-36.

30. Hsueh P-R, Huang L-M, Chen P-J, Kao C-L, Yang P-C. Chronological evolution of IgM, IgA, IgG and neutralisation antibodies after infection with SARS-associated coronavirus. Clin Microbiol Infect. 10, 2004, 1062-6.

31. Rokni M, Ghasemi V, Tavakoli Z. Immune responses and pathogenesis of SARSCoV-2 during an outbreak in Iran: Comparison with SARS and MERS. Rev Med Virol 2020.

32. Zhang B. Treatment with convalescent plasma for critically ill patients with SARSCoV-2 infection. Chest. 2020, 3057130577.

33. Ahn JY, Sohn Y, Lee SH. Use of convalescent plasma therapy in two covid-19 patients with acute respiratory distress syndrome in Korea. J Korean Med Sci. 35 (14), 2020, e149.
34. Ye Mingxiang, Fu Dian, Ren Yi. Treatment with convalescent plasma for COVID-19 patients in Wuhan, China. J Med Virol. 2020.

35. Shen C, Wang Z, Zhao F. Treatment of 5 critically III patients with COVID-19 with convalescent plasma. JAMA. 323(16), 2020, 1582-1589.

36. Karthick R, Krishnasamy N, Arunkumar R. Convalescent plasma transfusion for the treatment of COVID-19, Systematic review. J Med Virol 2020

37. Luke TC. Hark back: passive immunotherapy for influenza and other serious infections. Critical Care Medicine. 38(4), 2010, e66-e73.

38. Gajic 0 . Transfusion-related acute lung injury in the critically ill: prospective nested case-control study. American Journal of Respiratory and Critical Care Medicine. 176(9), 2007, 886891.

39. Lu CL, Murakowski DK, Bournazos S. Enhanced clearance of HIV-1-infected cells by broadly neutralizing antibodies against HIV-1 in-vivo. Science. 352, 2016, 1001-04.

40. Qing-Lei Zeng, Zu-Jiang Yu, Jian-Jun Gou,Guang-Ming Li,ShuHuan Ma.Effect of Convalescent Plasma Therapy on Viral Shedding and Survival in Patients With Coronavirus Disease 2019, 2020, 1-6.

41. Cunningham A.C, Goh H.P. and Koh D. Treatment of COVID19, old tricks for new challenges. Critical Care. 24, 2020, 91.

Source of Support: None declared.

Conflict of Interest: None declared.

For any question relates to this article, please reach us at: editor@globalresearchonline.net

New manuscripts for publication can be submitted at: submit@globalresearchonline.net and submit_ijpsrr@rediffmail.com 